UCLA $/ 95 /$ TEP $/ 13$

\title{
GENERAL 2+1 DIMENSIONAL EFFECTIVE ACTIONS AND SOLITON SPIN FRACTIONALIZATION
}

\author{
Eric D'Hoker * \\ dhoker@physics.ucla.edu \\ Department of Physics and Astronomy \\ University of California at Los Angeles \\ Los Angeles, CA 90024, USA
}

\begin{abstract}
We propose actions for non-linear sigma models on cosets $G / H$ in $2+1$ dimensions that include the most general non-linear realizations of Chern-Simons terms. When $G$ is simply connected and $H$ contains $r$ commuting $\mathrm{U}(1)$ factors, there are $r$ different topologically conserved charges and generically $r$ different types of topological solitons. Soliton spin fractionalizes as a function of the Chern-Simons couplings, with independent spins associated to each species of soliton charge, as well as to pairs of different charges. This model of soliton spin fractionalization generalizes to arbitrary $G / H$ a model of Wilczek and Zee for one type of soliton.
\end{abstract}

* Research is supported in part by National Science Foundation grant PHY-92-18990. 
A complete characterization of invariant effective actions for Goldstone boson fields due to the spontaneous breakdown of a symmetry group $G$ to a subgroup $H$ was recently obtained in Ref. [1]. In addition to manifestly $G$-invariant contributions arising from invariant Lagrangian densities, there may in general be special terms in the action that do not correspond to invariant Lagrangian densities. These special actions were shown to be in one to one correspondence with non-trivial generators of the de Rham cohomology of $G / H$. They comprise the Wess-Zumino-Witten terms [2] but may also receive contributions involving Goldstone-Wilczek currents [3]. For $2+1$ dimensional space-time $M_{3}$, the special classical actions are given by

$$
S[\pi]=\int_{B_{4}} \Omega(\tilde{\pi})
$$

where $\Omega(\tilde{\pi})$ is a cohomology generator of degree 4 belonging to $H^{4}(G / H ; \mathbf{R}), B_{4}$ is a four ball with boundary $M_{3}$ and $\tilde{\pi}$ is a field on $B_{4}$ that continuously interpolates between the original Goldstone field $\pi(x)$ and the field 0 .

In this letter, we determine explicitly the most general form of special classical actions in $2+1$ dimensions and the quantization conditions for the associated coupling constants. Furthermore, when $G$ is simply connected and $H$ contains $r$ independent commuting $U(1)$ factors, there are $r$ different topologically conserved currents with associated charges $Q_{i}$ and $r$ different species of topological solitons. We derive Bogomolnyi bounds on the masses and show that in general soliton spin fractionalizes as a function of the non-linear ChernSimons coupling constants and the soliton charges. In addition to a fractional spin for each species of soliton charge, there are also spin factors associated with pairs of different charges. This model of soliton spin fractionalization generalizes to arbitrary $G / H$ the models considered for $G / H=S^{2}$ by Wilczek and Zee [4,5], and for $G / H=\mathbf{C} P^{N}$ in [7], where only one species of solitons occurs. (See also [8] for applications in condensed matter physics.)

We begin by constructing the most general special actions of (1). All cohomology generators were constructed in Ref. [9], and may be conveniently expressed in terms of the components of the Maurer-Cartan connection defined by $U^{-1} d U=\theta^{A} T^{A}=\theta_{\mu}^{A} T^{A} d x^{\mu}$. Here, $U(\pi)$ parametrizes the Goldstone field $\pi(x)$ and $T^{A}$ are the representation matrices of $\mathcal{G}$ in the representation ${ }^{\dagger}$ of $U$. The most general cohomology generator of degree 4

${ }^{\dagger}$ Indices $A, a$ and $\alpha$ run over the generators of the Lie algebra $\mathcal{G}$ of $G$, the Lie algebra $\mathcal{H}$ of $H$ and the complement $\mathcal{M}$ of $\mathcal{H}$ in $\mathcal{G}$ respectively, and $f_{\alpha \beta \gamma}$ denotes the structure constants of $H$. 
takes the form $\Omega(\tilde{\pi})=\Omega_{0}(\tilde{\pi})+\Omega_{1}(\tilde{\pi})+\Omega_{2}(\tilde{\pi})$ with

$$
\begin{aligned}
& \Omega_{1}(\tilde{\pi})=\sum_{\alpha, \beta=1}^{\operatorname{dimH}} m_{\alpha \beta} W_{\alpha} W_{\beta} \quad W_{\alpha}=d \theta_{\alpha}+\frac{1}{2} f_{\alpha \beta \gamma} \theta_{\beta} \theta_{\gamma} \\
& \Omega_{2}(\tilde{\pi})=\sum_{\sigma_{l}} \mu_{1}^{\left\{\sigma_{l}\right\}} \theta^{\sigma_{1}} \theta^{\sigma_{2}} \theta^{\sigma_{3}} \theta^{\sigma_{4}}+\sum_{\sigma_{1}, \sigma_{2}, i} \mu_{2}^{\left(\sigma_{l}, i\right)} \theta^{\sigma_{1}} \theta^{\sigma_{2}} W_{\alpha_{i}}+\sum_{\sigma, \rho} \mu_{3}^{(\sigma, \rho)} \theta^{\sigma} \Omega_{3}^{(\rho)}
\end{aligned}
$$

Here, $\Omega_{0}(\tilde{\pi})$ is a generator with trivial cohomology which is the exterior derivative of a manifestly invariant 3 -form, $W_{\alpha}$ is the field strength of the $\mathcal{H}$-valued connection $V$ with components $V_{\alpha}=\theta_{\alpha}$ and $m_{\alpha \beta}$ is a symmetric tensor on $\mathcal{H}$, invariant under the adjoint action of $\mathcal{H}$. The differential forms $\Omega_{1}(\tilde{\pi})$ generalize the well-known $\operatorname{tr} F \tilde{F}$ term in four dimensions, and may similarly be written as total exterior derivatives of generalized ChernSimons terms $[10,11]$. The contribution $\Omega_{2}(\tilde{\pi})$ arises when cohomology generators of degree 1 , denoted by $\theta^{\sigma}, \sigma=1, \cdots, \operatorname{dim} H^{1}(G / H ; \mathbf{R})$, are also present; this happens only when $G / H$ is not simply connected. In the expression for $\Omega_{2}(\tilde{\pi}), \mu_{1}, \mu_{2}$ and $\mu_{3}$ are arbitrary coefficients, anti-symmetric in their $\sigma$ indices, and $\sigma, \alpha_{i}$ and $\rho$ running over the generators of $H^{1}(G / H ; \mathbf{R}), H^{2}(G / H ; \mathbf{R})$ and $H^{3}(G / H ; \mathbf{R})$ respectively.

The subgroup $H$ admits the following decomposition into $q$ connected, simply connected, simple Lie groups $H_{p}, p=1, \cdots, q$, times $r$ commuting $U(1)$ factors and a finite discrete subgroup $D$ :

$$
H=H_{1} \times \cdots \times H_{q} \times U(1)^{r} \times D
$$

The invariant tensor $m_{\alpha \beta}$ decomposes under this product and reduces to the Cartan-Killing form on the simple factors $H_{p}$. As a result, the effective actions in Eq. (1) may be rewritten directly in $2+1$ dimensional space-time, with $S[\pi]=S_{0}[\pi]+S_{1}[\pi]+S_{2}[\pi]$ and

$$
\begin{aligned}
& S_{1}[\pi]=\sum_{p=1}^{q} m_{2}^{p} \int_{M_{3}}\left(V_{\alpha}^{(p)} d V_{\alpha}^{(p)}+\frac{2}{3} f_{\alpha \beta \gamma} V_{\alpha}^{(p)} V_{\beta}^{(p)} V_{\gamma}^{(p)}\right)+\sum_{i, j=1}^{r} m_{1}^{i j} \int_{M_{3}} V_{\alpha_{i}} d V_{\alpha_{j}} \\
& S_{2}[\pi]=\sum_{\sigma_{l}} \mu_{1}^{\left\{\sigma_{l}\right\}} \int_{M_{3}} \psi^{\sigma_{1}} \theta^{\sigma_{2}} \theta^{\sigma_{3}} \theta^{\sigma_{4}}+\sum_{i, \sigma_{l}} \mu_{2}^{i \sigma_{l}} \int_{M_{3}} \psi^{\sigma_{1}} \theta^{\sigma_{2}} W_{\alpha_{i}}+\sum_{\sigma, \rho} \mu_{3}^{\sigma \rho} \int_{M_{3}} \psi^{\sigma} \Omega_{3}^{(\rho)}
\end{aligned}
$$

Here, $S_{0}[\pi]$ contains only manifestly invariant contributions, such as kinetic terms with two derivative, Skyrme terms with four derivatives and possibly higher order derivative terms. (We shall classify terms with two derivatives shortly.) The contribution $S_{1}[\pi]$ results from substituting $\Omega[\tilde{\pi}]$ of Eq. (2) into the general expression for the action in Eq. (1) and 
using the fact that $\Omega[\tilde{\pi}]$ is a total differential. These terms correspond to Chern-Simons actions [10] evaluated on composite gauge fields $V_{\alpha}$, and we shall name them non-linear Chern-Simons terms. The contribution $S_{2}[\pi]$ is obtained from $\Omega_{2}(\tilde{\pi})$ in $(3)$ with the help of the angles $\psi^{\sigma}$ that parametrize the homology cycles dual to $\theta^{\sigma}=d \psi^{\sigma}$.

The actions $S_{1}[\pi]$ and $S_{2}[\pi]$ are not in general gauge invariant, even though their contributions to the Euler-Lagrange equations are covariant. At the quantum level, the action is required to be invariant only up to additive shifts which are integer multiples of $2 \pi i$. This condition in general requires certain of the coupling constants $m_{1}, m_{2}, \mu_{1}$, $\mu_{2}$ or $\mu_{3}$ to be quantized in integer multiples of some basic units [10,11]. Precisely which of these coupling constants must be quantized depends upon the topology of space-time $M_{3}$. If space-time is compactified to a sphere $S^{3}$, only the Chern-Simons couplings $m_{2}^{p}$ corresponding to the simple factors $H_{p}$ must be quantized, while the couplings $m_{1}^{i j}, \mu_{1}$, $\mu_{2}$ and $\mu_{3}$ remain unquantized. On the other hand, if space-time is $M_{3}=S^{2} \times S^{1}$, the couplings $m_{1}$ must be quantized, while the couplings $m_{2}$ remain unquantized. More generally, the couplings $m_{1}$ and $\mu_{i}$ must be quantized whenever $M_{3}$ is not simply connected, while $m_{2}$ must be quantized if $\pi_{3}\left(M_{3}\right) \neq 0$.

For simplicity, we shall henceforth assume $G$ simply connected, $H$ connected (with the discrete subgroup $D=1)$, so that $G / H$ is simply connected, $H^{1}(G / H ; \mathbf{R})=0$ and the contribution $S_{2}[\pi]$ vanishes identically. As a result, $H^{2}(G / H ; \mathbf{Z}) \sim \pi_{2}(G / H)=\pi_{1}(H)=$ $\oplus_{r} \mathbf{Z}$, and there exist $r$ independent topologically conserved currents which are proportional to the $U(1)^{r}$ field strengths $W_{\alpha_{l}}=d V_{\alpha_{l}}$. Their associated conserved topological charges are given by

$$
Q_{i}=\int_{M_{2}} W_{\alpha_{i}} \quad i=1, \cdots, r
$$

Gauging these topological currents yields an equivalent representation of the special action $S_{1}$ of (4), considered here just for $m_{2}=0$. To see this, we consider

$$
\tilde{S}_{1}[\pi, B]=2 \sum_{i=1}^{r} \int_{M_{3}} B^{i} W_{\alpha_{i}}-\sum_{i, j=1}^{r} g_{i j} \int_{M_{3}} B^{i} d B^{j}
$$

The associated Euler-Lagrange equation for $B^{i}$ can be solved explicitly in terms of $V_{\alpha_{j}}$ and arbitrary gauge transformation functions $\omega^{i}$ :

$$
B_{0}^{i}=\sum_{j=1}^{r}\left(g^{-1}\right)^{i j} V_{\alpha_{j}}+d \omega^{i}
$$


Thus, $B^{i}$ is not a dynamical field, even though it enters (6) with derivatives. It may be eliminated from $\tilde{S}_{1}$, yielding precisely $S_{1}[\pi]$ (again for $m_{2}=0$ ) provided $g_{i j}$ is the inverse matrix of $m_{1}^{i j}$. $\tilde{S}_{1}$ may be viewed as an ordinary (linear) Chern-Simons interaction for gauge fields coupled to topological currents.

Topological solitons, labelled by $r$ independent charges $Q_{i}, i=1, \cdots, r$ arise in these models. Mathematically, these solitons are identical to $1+1$ dimensional instantons on coset spaces $G / H[12]$. Physically, they strongly resemble 't Hooft-Polyakov magnetic monopoles in $3+1$ dimensions [13], produced by the spontaneous breaking of a semi-simple group $G$ to a subgroup $H$ containing $r$ commuting (electro-magnetic) $U(1)$ factors. (See also [14].) The precise shape of the soliton solution depends upon the nature of the action $S_{0}[\pi]$ and on the group theory of $G / H$. Here, we shall consider only the two-derivative kinetic contributions to $S_{0}[\pi]$, but higher order terms are easily included. As the properties of solitons depend primarily on the $U(1)$ factors in $H$, we omit discussion of the $m_{2}$ term in $S_{1}$.

We evaluate the following soliton quantum numbers : topological charges, mass and spin. We denote the Cartan generators of $\mathcal{G}$ by $h_{i}, i=1, \cdots, \operatorname{rank} G$, of which $i=1, \cdots, r$ generate $U(1)^{r}$ in $\mathcal{H}$. Roots $a$ are associated with generators $T_{a}$ with $\left[h_{i}, T_{a}\right]=a_{i} T_{a}$ and $\left[T_{a}, T_{-a}\right]=\sum_{i} a_{i} h_{i}$. The conserved topological charges can be expressed as a sum over positive roots

$$
Q_{i}=-i \sum_{a>0} a_{i} \int_{M_{2}} d^{2} x \epsilon^{m n} \theta_{m}^{a} \theta_{n}^{-a} \quad i=1, \cdots, r
$$

They take on integer values provided $\theta_{m}^{a}$ falls off sufficiently rapidly as $|\vec{x}| \rightarrow \infty$. The most general kinetic action with just two derivatives can be expressed as

$$
S_{0}[\pi]=\sum_{a, b} \frac{1}{2} K_{a b} \int_{M_{3}} d^{3} x \theta_{\mu}^{a} \theta_{\mu}^{b}
$$

Here, $K_{a b}$ is symmetric with positive eigenvalues, and $U(1)^{r}$ invariance requires $a_{i}+b_{i}=0$ for all $i=1, \cdots, r$. When $r<\operatorname{rank} G$, the sum over $a$ and $b$ may in principle also contain terms from Cartan generators $h_{i}, i=r+1, \cdots$, rank $G$ which are not in $U(1)^{r}$. In particular, when non-trivial simple components $H_{p}$ are present in $H$ in addition to $U(1)^{r}$, $K_{a b}$ should be invariant under $H_{p}$. Henceforth, to simplify the discussion, we shall assume that $K_{a b}$ vanishes unless $a$ and $b$ are opposite roots (This is always the case when rank $G$ $=\operatorname{rank} H$.) and $K_{a-a} \geq 0$ for all positive roots $a$.

The masses of topological solitons obey Bogomolnyi type bounds [15] at the classical level. To derive those, we start from the following identity, valid for a fixed root $T_{a}$. 
$(m, n=1,2$ run over space coordinates only.)

$$
\int_{M_{2}} d^{2} x \theta_{m}^{a} \theta_{m}^{-a}=\frac{1}{2} \int_{M_{2}} d^{2} x\left(\theta_{m}^{a} \pm i \epsilon_{m n} \theta_{n}^{a}\right)\left(\theta_{m}^{-a} \mp i \epsilon_{m p} \theta_{p}^{-a}\right) \pm i \int_{M_{2}} d^{2} x \epsilon_{m n} \theta_{m}^{a} \theta_{n}^{-a}
$$

Let $\vec{\lambda}$ be a vector with components $\lambda_{i}$ which is independent of the roots $a$ and such that for all positive roots,

$$
K_{a-a}=\vec{\lambda} \cdot \vec{a}+N_{a} \quad N_{a} \geq 0 \quad \vec{\lambda} \cdot \vec{a} \geq 0
$$

(The vector $\vec{\lambda}$ is naturally proportional to a vector in the lattice dual to the roots $\vec{a}$, which can be viewed as a highest weight vector of some irreducible representation of $G$.) For a given set of constants $K_{a-a}$, there may be several possible choices for $\vec{\lambda}$. For each choice, we obtain the following bound on the energy $E[\pi]$ of any static configuration $\pi(x)$ :

$$
\begin{aligned}
E[\pi] & =\sum_{a>0} N_{a} \int_{M_{2}} d^{2} x \theta_{m}^{a} \theta_{m}^{-a}+\frac{1}{2} \sum_{a>0} \vec{\lambda} \cdot \vec{a} \int_{M_{2}} d^{2} x\left|\theta_{m}^{a} \pm i \epsilon_{m n} \theta_{n}^{a}\right|^{2} \mp \vec{\lambda} \cdot \vec{Q} \\
& \geq|\vec{\lambda} \cdot \vec{Q}|
\end{aligned}
$$

The lower bound can be saturated under the following conditions on the various roots : if $\mp \vec{\lambda} \cdot \vec{Q}>0$, then

$$
\begin{cases}N_{a}=0 & \theta_{m}^{a} \pm i \epsilon_{m n} \theta_{n}^{a}=0 \\ N_{b} \neq 0 & \theta_{m}^{b}=0\end{cases}
$$

Thus, the Maurer-Cartan form $\theta_{m}$ lives in a subalgebra of $\mathcal{G}$ in which all roots have $N_{a}=0$.

Solutions may be found explicitly in terms of analytic functions using techniques of $[12,14]$. The simplest case is when the soliton lives in an $S U(2)$ subgroup of $G$ which contains a generator of the Abelian $U(1)$ factor $U(1)^{r}$ in $H$. Then, the $S U(2)$ subalgebra is spanned by a Cartan generator $h \in U(1)^{r}$ and by the roots $T_{c}$ and $T_{-c}$. The MaurerCartan form for a configuration that satisfies the Bogomolnyi bound vanishes for all roots except $c$ and $-c$, and we have

$$
\theta_{m}=\theta_{m}^{h} h+\theta_{m}^{c} T_{c}+\theta_{m}^{-c} T_{-c}
$$

The Cauchy-Riemann equations of (13) reduce to $\theta_{\bar{z}}^{c}=0$, or equivalently $\theta_{z}^{-c}=0$. This equation is easily solved as follows :

$$
U(z, \bar{z})=\exp \left\{f^{c}(z) T_{c}\right\} \cdot \exp \left\{f^{-c}(z, \bar{z}) T_{-c}\right\} \cdot \exp \left\{f^{h}(z, \bar{z}) h\right\}
$$


Here $f^{-c}$ and $f^{h}$ are arbitrary functions of $z$ and $\bar{z}, f^{c}$ is holomorphic in $z$, and one imposes the additional requirement that $U(z, \bar{z})$ be in $\mathcal{G}$. For $S U(2)$ in the fundamental representation for example, we have

$$
U(z, \bar{z})=\left(\begin{array}{cc}
1 & 0 \\
i f(z) & 1
\end{array}\right) \cdot\left(\begin{array}{cc}
\rho(z, \bar{z}) & i \overline{f(z)} \rho(z, \bar{z}) \\
0 & \rho^{-1}(z, \bar{z})
\end{array}\right) \cdot\left(\begin{array}{cc}
e^{i \varphi(z, \bar{z})} & 0 \\
0 & e^{-i \varphi(z, \bar{z})}
\end{array}\right)
$$

with the requirement that $\left(1+|f(z)|^{2}\right) \rho^{2}(z, \bar{z})=1$. The corresponding soliton solutions satisfy $Q_{i_{0}}=1$ and $Q_{i}=0$ for $i \neq i_{0}$, and are elementary solitons with the lowest possible non-trivial charge assignment.

The position of the Bogomolnyi soliton inside $G / H$ may however be more general than considered above. It is determined by the position of the homology 2 cycle in $G / H$ dual to the closed invariant 2 forms $W_{\alpha_{i}}$. For example, in the case of $G / H=S U(3) / U(1)$ we take $H=U(1)$ the subgroup generated by the Lie algebra generator $\lambda_{8} \sim \operatorname{diag}\left(\begin{array}{lll}1 & 1 & -2\end{array}\right)$, which does not belong to any $S U(2)$ subalgebra of $S U(3)$. There is only a single conserved current, $W_{\lambda_{8}}$, which uniquely determines the soliton charge. Clearly, $W_{\lambda_{8}}$ is invariant under local $S U(2)$ right multiplications of $U$ that commute with $\lambda_{8}$. As a result, $W_{\lambda_{8}}$ projects down to a well-defined closed 2-form on the coset of $G / H$ by $S U(2)$ which is $\mathbf{C} P^{2}=S U(3) / S(U(2) \times U(1))$. On this space, $W_{\lambda_{8}}$ is just the Kähler class, whose structure is well-known [16]. More generally, when $r<\operatorname{rank} G$, one may use the methods that generalize our discussion for $S U(3)$, by forming cosets of $G / H$ by subgroups of $G$ that leave the relevant $W_{\alpha_{i}}$ invariant, until one arrives at a space $G / H^{\prime}$ with $\operatorname{rank} H^{\prime}=\operatorname{rank} G$. These spaces can be viewed as classifying spaces [17] for the second cohomology classes of $G / H$.

The Bogomolnyi bounds guarantee that the solitons obey the field equations, but they allow for the possibility that some solutions correspond to multi-particle configurations. In general, the analysis of stability is complicated by the arbitrariness of the kinetic energy coefficients $K_{a-a}$ in (9), upon whose values the stability questions depend. In the simplest case, rank $G=r$, elementary solitons with $Q_{i_{0}}=1$ and $Q_{i}=0$ for $i \neq i_{0}$, are all stable. Interaction energies between different elementary solitons vanish when all $N_{a}=0$. Thus, all non-elementary configurations with non-negative charges $Q_{i}$ are multi-solitons composed of non-interacting elementary solitons. This property is very much analogous to that of multi-monopole configurations. Elementary solitons and anti-solitons (with negative elementary charge $Q_{i}$ ) presumably attract one another, but this conclusion does not follow from the Bogomolnyi bounds. 
The soliton spin was evaluated in $[4,5]$ for $G / H=S^{2}$ by considering the change in the action under a rotation of the soliton configuration. Generalization of this method to arbitrary $G / H$ is straightforward only when the solitons are embedded into $G$ in a simple way, as discussed above. Parametrizations of solitons in more general embeddings however is cumbersome. Fortunately, a simple determination of soliton spin is available directly from current algebra [6] at the classical level, which we shall make use of here. We set $S_{2}[\pi]=0$ and $m_{2}=0$, so that the only remaining non-linear Chern-Simons action is the $m_{1}$ term in $S_{1}[\pi]$. All other contributions to the effective action contained in $S_{0}[\pi]$ are manifestly invariant. To fix ideas, we may think of this action as just the kinetic term, given in (9). Following [6], the definition of spin is through the stress tensor

$$
J=\epsilon_{m n} \int_{M_{2}} d^{2} x x^{m} T^{0 n}
$$

The stress tensor $T^{\mu \nu}$ is constructed as the variation of the action under the cahnge of an auxiliary background space-time metric tensor, and thus receives no contribution from the topological part of the action, $S_{1}[\pi]$. We find

$$
T^{0 n}=\partial_{n} \pi^{a} \Pi_{0}^{a} \quad \Pi_{0}^{a}=\frac{\partial \mathcal{L}_{0}}{\partial\left(\partial_{0} \pi^{a}\right)}
$$

where $\Pi_{0}^{a}(x)$ is the part of the momentum canonically conjugate to $\pi^{a}$, arising only from the action $S_{0}$ with Lagrangian density $\mathcal{L}_{0}$. It is related to the full canonical momentum $\Pi^{a}$, by the addition of a term involving only the action $S_{1}$ :

$$
\Pi^{d}(x)=\Pi_{0}^{d}(x)+\sum_{i, j} m_{1}^{i j} \epsilon_{m n} f^{\alpha_{i} b c} M^{b d}(\pi(x)) \theta_{m}^{c}(x) V_{n}^{\alpha_{j}}(x)
$$

where $M^{b d}$ is defined by $\theta_{m}^{b}=M^{b d} \partial_{m} \pi^{d}$. One proceeds by expressing the composite gauge field $V_{n}^{\alpha_{j}}$ in terms of the topologically conserved current $W^{\alpha_{j}}$. It is then straightforward to work out the formula for the contribution to the spin arising from the non-linear ChernSimons term $S_{1}$, and we find

$$
J=\int_{M_{2}} d^{2} x \epsilon_{m n} x_{m} \Pi^{a}(x) \partial_{n} \pi^{a}(x)-\frac{1}{2 \pi} \sum_{i, j} m_{1}^{i j} Q_{i} Q_{j}
$$

The first term on the right hand side is the standard spin, which in canonical quantization receives only integer contributions. The second term arises purely from the non-linear Chern-Simons term. While the values of $Q_{i}$ are integers for configurations that fall off 
sufficiently fast at spatial infinity, the coupling constants $m_{1}^{i j}$ do not have to be quantized in general. As a result, the non-linear Chern-Simons contribution to the spin $J$ is in general fractional. When there is only a single topologically conserved charge, we recover the expression of [6]. However, for several different conserved charges, the off diagonal entries in $m_{1}^{i j}$ contribute and add spin from the presence of several different charges $Q_{i}$.

\section{Acknowledgements}

I have benefitted from helpful conversations with Roman Jackiw, Jeff Rabin, Terry Tomboulis, Steven Weinberg and Tony Zee.

\section{References}

[1] E. D'Hoker and S. Weinberg, Phys. Rev. D50 (1994) 605.

[2] J. Wess and B. Zumino, Phys. Lett. 37B (1971) 95; E. Witten, Nucl. Phys. B223 (1983) 422, 433.

[3] J. Goldstone and F. Wilczek, Phys. Rev. Lett. 47 (1981) 986; E. D'Hoker and J. Goldstone, Phys. Lett. 158B (1985) 429.

[4] F. Wilczek and A. Zee, Phys. Rev. Lett. 51 (1983) 2250; Y.S. Wu and A. Zee, Phys. Lett. 147B (1984) 325.

[5] F. Wilczek, Fractional Statistics and Anyon Superconductivity, World Scientific, Singapore, 1990; E. Fradkin, Field Theories of Condensed Matter Systems, Addison Wesley, New York, 1991; W.J. Zakrzewski, Low Dimensional Sigma Models, Adam Hilger, London 1989.

[6] M.J. Bowick, D. Karabali, L.C.R. Wijewardhana, Nucl. Phys. B271 (1986) 417.

[7] N.K. Pak, Phys. Lett. 260B (1991) 377; G. Ferretti and S.G. Rajeev, Phys. Rev. Lett. 69 (1992) 2033; G. Ferretti, S.G. Rajeev and Z. Yang, Int. J. Mod. Phys. A7 (1992) 7989; K. Fujii, Commun. Math. Phys. 162 (1994) 273; K. Fujii, J. Math. Phys. 36 97; S. Forte, Rev. Mod. Phys. 64 (1992) 193; A.P. Balachandran, M. Bourdeau and S. Jo, Int. J. Mod. Phys. A5 (1990) 2423.

[8] Y.-S. Wu, Phys. Rev. Lett. 52 (1984) 2103; D.P. Arovas, J.R. Schrieffer, F. Wilczek and A. Zee, Nucl. Phys. B251 (1985) 117; D.-H. Lee and C.L. Kane, Phys. Rev. Lett. 64 (1990) 1313; S.L. Sondhi, A. Karlhede, S.A. Kivelson and E.H. Rezayi, Phys. Rev. B47 (1993) 16419; K. Moon, H. Mori, K. Yang, S.M. Girvin, A.H. MacDonald, 
L. Zheng and D. Yoshioka, Phys. Rev. 51 (1995) 5138; C. Nayak and F. Wilczek, PUPT 1540, IASSNS-HEP 95/35 preprint, May 1995.

[9] E. D'Hoker, "Invariant Effective Actions, Cohomology of Homogeneous Spaces and Anomalies", UCLA/95/TEP/5 preprint (1995), hep-th/9502162, accepted for publication in Nucl. Phys. B.; E. D'Hoker, "Invariant Effective Actions and Cohomology", UCLA/95/TEP/11 preprint (1995), hep-th/9505109.

[10] S. Deser, R. Jackiw and S. Templeton, Phys. Rev. Lett. 48 (1982) 975; Ann. Phys. (NY) 140 (1982) 372; J. Schonfeld, Nucl. Phys. B185 (1981) 157; W. Siegel, Nucl. Phys. B (1982).

[11] R. Jackiw, in Current Algebra and Anomalies, by S.B. Treiman, R. Jackiw, B. Zumino and E. Witten, Princeton Univ. Press, 1989.

[12] A. Belavin and A.M. Polyakov, JETP Lett. 22 (1975) 245; V. Golo and A. Perelemov, Phys. Lett. 79B (1978) 112; A. D'Adda, P. Di Vecchia and M. Lüscher, Nucl. Phys. B146 (1978) 63; A. Perelemov, Phys. Rep. 146 (1987) 135.

[13] G. 't Hooft, Nucl. Phys. B79 (1974) 276; A.M. Polyakov, JETP Lett. 20 (1974) 194.

[14] R. Jackiw, K. Lee, E. Weinberg, Phys. Rev. D42 (1990) 3488; R. Jackiw and S.-Y. Pi, Phys. Rev. Lett. 64 (1990) 2969.

[15] E. Bogomolnyi, Sov. J. Nucl. Phys. 24 (1976) 449.

[16] B.A. Dubrovin, A.T. Fomenko and S.P. Novikov, Modern Geometry and Applications, Springer Verlag, 1990.

[17] N. Steenrod, The Topology of Fibre Bundles, Princeton U. Press, 1951. 